\title{
A Decision Support Approach for Group Decision Making under Risk and Uncertainty
}

\author{
Jiawei $\mathrm{Li}^{1}$, Graham Kendall ${ }^{1}$, Simon Pollard ${ }^{2}$, Emma Soane ${ }^{3}$, Gareth Davies ${ }^{2}$, Ruibin Bai ${ }^{4}$ \\ 1. School of Computer Science, University of Nottingham, Nottingham, UK, NG8 1BB \\ E-mail: jwl@cs.nott.ac.uk (J. Li),gxk@cs.nott.ac.uk (G. Kendall) \\ 2. School of Applied Sciences, Cranfield University, Cranfield, MK43 0AL \\ E-mail: s.pollard@cranfield.ac.uk (S. Pollard), g.davies@cranfield.ac.uk (G. Davies)
}

3. Department of Management, London School of Economics, London, WC2A 2AE

E-mail: E.C.Soane@1se.ac.uk (E. Soane)

4. Division of Computer Science, University of Nottingham Ningbo, China 315100

E-mail: ruibin.bai@nottingham.edu.cn (R. Bai)

\begin{abstract}
Cost-benefit analysis has long been used in decision making about public health and security. Frequently, risk and uncertainty are involved, and benefit and cost are not evenly shared by all stakeholders in the activities where public welfare is concerned. The result of cost-benefit analysis may be controversial because it does not consider the conflict of interest among the stakeholders. In this paper, we propose a decision support approach that allows individual agents to make their own evaluations of benefit, cost and risk over available alternatives. Individual beliefs with respect to the alternatives will then be aggregated to form a group decision. This approach can also be used to integrate the cost benefit analysis into risk assessment. An application to this group decision making, considering the disposal of dead animals, is given.
\end{abstract}

Key Words: Decision support approach, Cost-benefit analysis, Uncertainty, risk, Group decision making, Evidence support logic, Expected utility

\section{INTRODUCTION}

Cost-benefit analysis (CBA) was originally used to evaluate the desirability of governmental intervention in markets, and has now been used in many areas of public decision making such as transportation, environment, health care, and safety. As an economic approach, it shows the monetary values of benefit and cost of the targeted activity. This analysis is required in many decision making processes.

However, the role of $\mathrm{CBA}$ in decision making is controversial when public welfare is concerned, especially when risk and uncertainty are involved. Most researchers and regulators agree that $\mathrm{CBA}$ is neither sufficient nor necessary for the final decisions because of its weaknesses ${ }^{[1,2]}$. Firstly, in most cases where public welfare is concerned, benefit and cost are not evenly shared by all stakeholders. The stakeholders that pay for the expenditures or bear the risk may not necessarily receive sufficient benefit from the activity. CBA does not take into account conflict of interest among stakeholders. Secondly, not all the important factors in decision making can be quantified and CBA cannot deal with this uncertainty. Thirdly, economic payoff is not the primary objective in many circumstances, especially when there is a risk of hazard. Therefore, there are still challenges in order to integrate the result of CBA into the framework of risk assessment.

In practice, there is discussion, negotiation, and concession between decision maker and stakeholders, especially when the consequences are potentially serious. For example, a regulatory decision on the disposal of nuclear waste might involve the

This work is supported by EPSRC (Engineering and Physical Sciences Research Council) grant (Ref: EP/E017975/1). regulatory committee, the operators, the regulation advisors, scientists, government policy makers, etc. There would be lengthy discussions before a final decision could be made. No single agent has the power to make the final decision. It is actually a group decision and each stakeholder has his/her influence on it.

In this paper, we propose a new decision support approach that integrates evidence support logic and expected utility theory. With this approach, the agents involved in a group decision making process are allowed to express their utilities or evaluations over different alternatives. These utilities, or monetary values, are translated into individual beliefs with respect to the comparison of alternatives with respect to each other. The group decision will then be given by aggregating individual beliefs. The group decision shows how much each alternative is preferred.

\section{PRELIMINARY KNOWLEDGE}

\section{A. Evidence support logic}

Evidence support logic (ESL) is an information propagation approach developed from interval probability theory $[3,4,5]$. It has been applied in several fields of risk regulation ${ }^{[7,8]}$. ESL deals with multiple pieces of evidence from different sources that may overlap or conflict with one another, and it allows a degree of uncertainty in evidence.

A belief is expressed by a triple $(p, u, q)$ where $p$ and $q$ denote the probabilities that some evidence supports or refutes a proposition and $u$ is the residual uncertainty. There is always $p+q+u=1,0 \leq p, q \leq 1$, and $-1 \leq u \leq 1 . u=1$ 
denotes the state of absolute ignorance and $u<0$ means that there is possible conflict within the evidence.

ESL has an simple algorithm to aggregate multiple beliefs (or evidence). Suppose that $n$ beliefs about the same proposition are expressed as $\left(p_{i}, u_{i}, q_{i}\right), i=1, \ldots, n$. Each belief is given a value to denote its sufficiency or how much weight it contributes to the proposition. The sufficiency of a belief takes a value range from 0 to 1 . A greater sufficiency will result in evidence values being more influential in the aggregation.

Let $\left(p_{A}, u_{A}, q_{A}\right)$ denote the aggregated belief. It can be computed as follows.

$$
\begin{aligned}
p_{A}= & \sum_{i=1}^{n} w_{i} p_{i}-\sum_{\substack{i, j=1 \\
i<j}}^{n} \rho_{i j} \min \left(w_{i} p_{i}, w_{j} p_{j}\right) \\
+ & \sum_{\substack{i, j, k=1 \\
i<j<k}}^{n} \rho_{i j k} \min \left(w_{i} p_{i}, w_{j} p_{j}, w_{k} p_{k}\right) \\
& +\ldots+(-1)^{n-1} \rho_{1, \ldots, n} \min \left(w_{1} p_{1}, \ldots, w_{n} p_{n}\right)
\end{aligned}
$$

where, if $S=\{i, j, \ldots\}$,

$$
\rho_{S}=\frac{(1-D) \prod_{a \in S} w_{a} p_{a}}{\min _{a \in S}\left(w_{a} p_{a}\right)}+D
$$

Here $w_{i}$ is the weighting of the $i^{t h}$ evidence; $D$ is the dependency between the evidence $p_{i}, p_{j}, \ldots, p_{n}$.

The computation of $q_{A}$ is similar to (1).

$$
\begin{gathered}
q_{A}=\sum_{i=1}^{n} w_{i} q_{i}-\sum_{\substack{i, j=1 \\
i<j}}^{n} \rho_{i j} \min \left(w_{i} q_{i}, w_{j} q_{j}\right) \\
+\sum_{\substack{i, j, k=1 \\
i<j<k}}^{n} \rho_{i j k} \min \left(w_{i} q_{i}, w_{j} q_{j}, w_{k} q_{k}\right) \\
+\ldots+(-1)^{n-1} \rho_{1, \ldots, n} \min \left(w_{1} q_{1}, \ldots, w_{n} q_{n}\right)
\end{gathered}
$$

Where

$$
\rho_{S}=\frac{(1-D) \prod_{a \in S} w_{a} q_{a}}{\min _{a \in S}\left(w_{a} q_{a}\right)}+D
$$

$u_{A}$ can be determined by means of $p_{A}+q_{A}+u_{A}=1$ once we have the values of $p_{A}$ and $q_{A}$. Specially, when $n=2$ and beliefs 1 and 2 are independent, we have,

$$
\left\{\begin{array}{c}
p_{A}=w_{1} p_{1}+w_{2} p_{2}-\max \left(w_{1} p_{1}, w_{2} p_{2}\right) \min \left(w_{1} p_{1}, w_{2} p_{2}\right) \\
q_{A}=w_{1} q_{1}+w_{2} q_{2}-\max \left(w_{1} q_{1}, w_{2} q_{2}\right) \min \left(w_{1} q_{1}, w_{2} q_{2}\right)
\end{array}\right.
$$

\section{B. Expected utility theory}

Expected utility theory is an approach to deal with the problem of decision making under risk and uncertainty in economics ${ }^{[9,10]}$. As a quantitative approach, it also has application in regulatory decision making ${ }^{[11]}$. The axiomatic hypothesis of expected utility is that the decision maker knows the probabilities of all outcomes of the activities.

Suppose an activity may lead to several possible outcomes and each outcome can be expressed as a monetary value. Assume the decision maker has a complete, reflexive, transitive, and continuous evaluation over these monetary outcomes, or in other words, he/she possesses a von Neumann-Morgenstern utility function. Let $x$ be an outcome and let $X$ be the set of possible outcomes. Let $p$ be a simple probability measure on $X$, thus $p=\left(p\left(x_{1}\right), p\left(x_{2}\right), \cdots, p\left(x_{n}\right)\right)$ where $p\left(x_{i}\right)$ are probabilities of outcome $x_{i} \in X(i=1, \cdots, n)$ occurring. Note that there are finite elements $x \in X$ for which $p(x)>0$, and that $p\left(x_{i}\right) \geq 0$ for all $i=1, \cdots, n$ and $\sum_{i=1}^{n} p\left(x_{i}\right)=1$. The expected utility over the set of outcomes $X$ is expressed as,

$$
U(X)=\sum_{i=1}^{n} u\left(x_{i}\right) p\left(x_{i}\right)
$$

Expected utility theory can also be applied as a CBA approach in risk regulation where public welfare is concerned. Consider a scenario of decision making under risk (disposal of nuclear waste, for example) where the risk is the possible realisation of environmental hazard. Suppose that the hazard may lead to a loss of wealth $w_{N}-w_{A}$ (measured by a monetary value), where $w_{N}$ denotes the original wealth and $w_{A}$ the reduced wealth if the hazard has occurred. In order to keep the risk within the acceptable range, an amount of money $C$ is going to be invested. The objective of regulatory decision making is to find the optimal amount of investment that maximizes the public welfare.

Let $\gamma$ denote the possibility (or risk) of the occurrence of an accident. We assume the existence of a state-independent utility function of the regulator $u(w)$ defined over payoffs, thus:

$$
U(\gamma, C)=\gamma u\left(w_{A}-C\right)+(1-\gamma) u\left(w_{N}-C\right)
$$

Notice that $U(\gamma, C)$ represents the expected utility of the regulator over public wealth and that $\gamma$ is a function of $C$ in the above equation. It has been proved in ${ }^{[11]}$ that, when the decision maker is risk-neutral, the condition of optimal expenditure against risk is,

$$
\gamma^{\prime}=1 /\left(w_{N}-w_{A}\right)
$$

Under (6), the risk is reduced to the degree so that a further reduction needs much more expenditure and is therefore not economical. 
Note that the optimal expenditure is independent of individual utility in (6). If the parameters of $w_{N}, w_{A}$ and $\gamma(C)$ are from unique sources and thus remain the same among all the stakeholders, (6) holds for different risk-neutral decision makers. Arrow and Lind ${ }^{[12]}$ have indicated that the decision maker should behave in a risk- neutral fashion when public welfare is concerned. Under the assumption of risk-neutral, it is possible for the decision to be unanimous within a group of stakeholders. However, if the stakeholders are not all risk-neutral or cost and benefit are not evenly shared, (6) will not hold.

In the following section, we study an approach to deal with group decision making where risk and benefit may be unevenly shared and the decision makers may have their own utilities toward risk and uncertainty.

\section{GROUP DECISION-MAKING: A DECISION SUPPORT APPROACH}

In this section, we will establish a decision support model that considers multiple agents involved in group decision making under risk and uncertainty. Each agent has his/her cost-benefit estimation over a few alternatives. The objective of the model is to determine the group decision based on individual utilities and evidence support logic.

Suppose that there are $m$ agents faced with $n$ alternatives $\left\{x_{1}, x_{2}, \cdots, x_{n}\right\}$ and each agent has a von Neumann-Morgenstern utility or monetary cost-benefit estimation over all alternatives. Here, the von Neumann-Morgenstern utility is not necessarily the evaluation of his/her own payoff. When the issue of concern is about public welfare, it is the evaluation of public wealth.

Let $U_{i}\left(x_{j}\right)$ denote agent $i$ 's expected utility of alternative $x_{j}$ where $i=\{1, \cdots, m\}$ and $j=\{1, \cdots, n\}$. For each agent, we establish a set of beliefs, each of which denotes the comparison between two different alternatives. Let triple ( $p_{j k}^{i}, u_{j k}^{i}, q_{j k}^{i}$ ) denote the agent $i$ 's belief with respect to the hypothesis 'Alternative $x_{j}$ is preferred to alternative $x_{k}$.' where $j, k=1, \cdots n$ and $j \neq k$. For all $n$ alternatives, every agent has a complete set of beliefs that contains $\frac{1}{2} n(n-1)$

items, each of which denotes a comparison between two different alternatives. For example, when $n=2$, there is only one belief with respect to the hypothesis 'Alternative $x_{1}$ is preferred to alternative $x_{2}$. When $n=3$, each agent has three beliefs.

Each agent assigns a set of uncertainty values $q_{j k}$ to each belief, which denotes how much uncertainty the agent has about this belief.

The relationship between individual beliefs and utilities of alternatives is expressed by,

$$
p_{j k}^{i}-q_{j k}^{i}=\frac{U_{i}\left(x_{j}\right)-U_{i}\left(x_{k}\right)}{D_{i}}
$$

Where

$$
D_{i}=\operatorname{Max}\left\{U_{i}\left(x_{1}\right), \cdots, U_{i}\left(x_{n}\right)\right\}-\operatorname{Min}\left\{U_{i}\left(x_{1}\right), \cdots, U_{i}\left(x_{n}\right)\right\} \text {. }
$$

Note that $p_{j k}^{i}+u_{j k}^{i}+q_{j k}^{i}=1$ and $0 \leq p_{j k}^{i}, q_{j k}^{i} \leq 1, p_{j k}^{i}$ and $q_{j k}^{i}$ can be uniquely determined by the follows.

$$
\begin{gathered}
\text { When }-\left(1-u_{j k}^{i}\right) \leq \frac{U_{i}\left(x_{j}\right)-U_{i}\left(x_{k}\right)}{D_{i}} \leq 1-u_{j k}^{i}, \\
\left\{\begin{array}{c}
p_{j k}^{i}=\frac{1-u_{j k}^{i}}{2}+\frac{U_{i}\left(x_{j}\right)-U_{i}\left(x_{k}\right)}{2 D_{i}} \\
q_{j k}^{i}=\frac{1-u_{j k}^{i}}{2}-\frac{U_{i}\left(x_{j}\right)-U_{i}\left(x_{k}\right)}{2 D_{i}}
\end{array}\right.
\end{gathered}
$$

When

$$
\begin{gathered}
\frac{U_{i}\left(x_{j}\right)-U_{i}\left(x_{k}\right)}{D_{i}}<-\left(1-u_{j k}^{i}\right), \\
\left\{\begin{array}{c}
p_{j k}^{i}=0 \\
q_{j k}^{i}=1-u_{j k}^{i}
\end{array}\right. \\
\text { When } \frac{U_{i}\left(x_{j}\right)-U_{i}\left(x_{k}\right)}{D_{i}>\left(1-u_{j k}^{i}\right),} \\
\left\{\begin{array}{c}
p_{j k}^{i}=1-u_{j k}^{i} \\
q_{j k}^{i}=0
\end{array}\right.
\end{gathered}
$$

Each agent is assigned a weight $w_{i}$ range from 0 to 1 that denotes his/her power in the group decision. It acts as the sufficiency of belief in the process of aggregation of multiple beliefs. Then, multiple agents' beliefs can be aggregated by means of (1) and (2). The aggregated beliefs denote the group preference over all alternatives.

We give an example to illustrate how this approach is applied in the following section.

\section{An ApPLiCATion}

The livestock and poultry industry have to face the problem of disposing of diseased animal carcases. Today's environmental legislation does not allow arbitrary disposal because of the possibility of pollution of water and the spread of disease. In the UK the Department of Environment, Food and Rural Affairs (DEFRA) are responsible for coordinating the disposal of diseased animal car cases. DEFRA collaborates with experts from a number of different parties (e.g. health departments and their agencies, veterinary officials, environment agencies, emergency planners and other professional partners) to inform their decisions regarding the most appropriate disposal option.

In this case study, we consider a scenario where a number of poultry farms in a district still adopt the traditional on-farm approaches to bury or burn dead animals. This has led to public 
concern of health and security. A regulatory committee is about to make decision on whether to pass a regulation to restrict these disposal methods.

Five agents are involved in the decision making process: DEFRA (Agent 1), governmental officers (Agent 2), environmental experts (Agent 3), industrial representatives (Agent 4) and local resident representatives (Agent 5). Three alternatives are considered.

- A1: Burial (status quo).

- A2: Constructed disposal pit.

- A3: Incineration.

A1 is the current on-farm approach to disposing of dead animals. It may lead to a hazard to both other animals and human health. There is also a high potential for ground water contamination from both bacteria and nutrients. A2 reduces the risk of hazard to animal and human health when compared with A1. However, it still has the potential to contaminate underground water and it may be odorous. A3 is a viable alternative that reduces the risk of environmental hazard to the minimum. The disadvantage is its higher construction and maintenance costs.

Each agent shows their payoffs (that might be the result of CBA or just individual evaluation). The payoffs of the agents in the form of either expected utilities or monetary values are shown in Table 1.

TABLE I Payoffs of the agents

\begin{tabular}{c|c|c|c}
\hline & $\mathrm{A} 1$ & $\mathrm{~A} 2$ & $\mathrm{~A} 3$ \\
\hline Agent 1 & 0 & 50 & 100 \\
\hline Agent 2 & 0 & 50 & 40 \\
\hline Agent 3 & -100 & 60 & 100 \\
\hline Agent 4 & 0 & $£ 2,000$ & $-£ 10,000$ \\
\hline Agent 5 & -100 & -80 & 100 \\
\hline \hline
\end{tabular}

There are three hypothesises.

$H_{1}$ : Alternative $A_{1}$ is prefered to $A_{2}$.

$H_{2}$ : Alternative $A_{1}$ is prefered to $A_{3}$.

$H_{3}$ : Alternative $A_{2}$ is prefered to $A_{3}$.

With respect to these hypothesises, each agent $i$ has three beliefs $\left(p_{12}^{i}, u_{12}^{i}, q_{12}^{i}\right),\left(p_{13}^{i}, u_{13}^{i}, q_{13}^{i}\right)$ and $\left(p_{23}^{i}, u_{23}^{i}, q_{23}^{i}\right)$. According to (7), the values of the beliefs are computed as shown in Table 2, 3, and 4.

By assigning each agent a weight of 0.2 , the aggregated belief can be computed by means of (1) and (2).

TESLA is a decision support software based on information propagation methods ${ }^{[13]}$. It provides a graphical interface and evidence support logic algorithm. We use TESLA to deal with the computation and the graphical expression of multiple beliefs.
Table II Individual beliefs on $\mathrm{H}_{1}$

\begin{tabular}{c|c}
\hline \hline Belief & Values \\
\hline$\left(p_{12}^{1}, u_{12}^{1}, q_{12}^{1}\right)$ & $(0.15,0.2,0.65)$ \\
\hline$\left(p_{12}^{2}, u_{12}^{2}, q_{12}^{2}\right)$ & $(0,0.3,0.7)$ \\
\hline$\left(p_{12}^{3}, u_{12}^{3}, q_{12}^{3}\right)$ & $(0,0.2,0.8)$ \\
\hline$\left(p_{12}^{4}, u_{12}^{4}, q_{12}^{4}\right)$ & $(0.42,0,0.58)$ \\
\hline$\left(p_{12}^{5}, u_{12}^{5}, q_{12}^{5}\right)$ & $(0.25,0.4,0.35)$ \\
\hline \hline
\end{tabular}

Table III Individual beliefs on $\mathrm{H}_{2}$

\begin{tabular}{c|c}
\hline \hline Belief & Values \\
\hline$\left(p_{23}^{1}, u_{23}^{1}, q_{23}^{1}\right)$ & $(0.15,0.2,0.65)$ \\
\hline$\left(p_{23}^{2}, u_{23}^{2}, q_{23}^{2}\right)$ & $(0.5,0.2,0.3)$ \\
\hline$\left(p_{23}^{3}, u_{23}^{3}, q_{23}^{3}\right)$ & $(0.3,0.2,0.5)$ \\
\hline$\left(p_{23}^{4}, u_{23}^{4}, q_{23}^{4}\right)$ & $(1,0,0)$ \\
\hline$\left(p_{23}^{5}, u_{23}^{5}, q_{23}^{5}\right)$ & $(0,0.4,0.6)$ \\
\hline \hline
\end{tabular}

TABLE IV Individual beliefs on $\mathrm{H}_{3}$

\begin{tabular}{c|c}
\hline \hline Belief & Values \\
\hline$\left(p_{13}^{1}, u_{13}^{1}, q_{13}^{1}\right)$ & $(0,0,1)$ \\
\hline$\left(p_{13}^{2}, u_{13}^{2}, q_{13}^{2}\right)$ & $(0,0.3,0.7)$ \\
\hline$\left(p_{13}^{3}, u_{13}^{3}, q_{13}^{3}\right)$ & $(0,0,1)$ \\
\hline$\left(p_{13}^{4}, u_{13}^{4}, q_{13}^{4}\right)$ & $(0.92,0,0.08)$ \\
\hline$\left(p_{13}^{5}, u_{13}^{5}, q_{13}^{5}\right)$ & $(0,0.4,0.6)$ \\
\hline
\end{tabular}

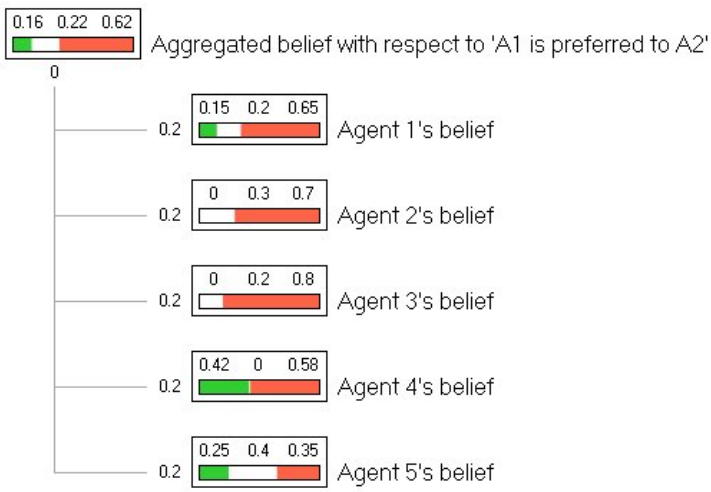

Figure I TESLA's interface.

The aggregated beliefs are computed as, $\left(p_{12}{ }^{A}, u_{12}^{A}, q_{12}^{A}\right)=(0.16,0.22,0.62)$ 


$$
\begin{aligned}
& \left(p_{23}{ }^{A}, u_{23}^{A}, q_{23}^{A}\right)=(0.39,0.2,0.41) \\
& \left(p_{13}{ }^{A}, u_{13}^{A}, q_{13}^{A}\right)=(0.18,0.14,0.68)
\end{aligned}
$$

The aggregated beliefs show that both alternatives A2 and $\mathrm{A} 3$ are preferred to alternative $\mathrm{A} 1$, and alternatives $\mathrm{A} 3$ is slightly preferred to alternative A2.

Figure II, III and IV show the ratio plots in which both individual beliefs and the aggregated beliefs are illustrated. The horizontal axis indicates the percentage uncertainty in the evidence and the vertical axis indicates the ratio of value for to value against. In Figure 2, all beliefs lie below the horizontal axis, which shows a consensus on 'A2 is better than A1'.

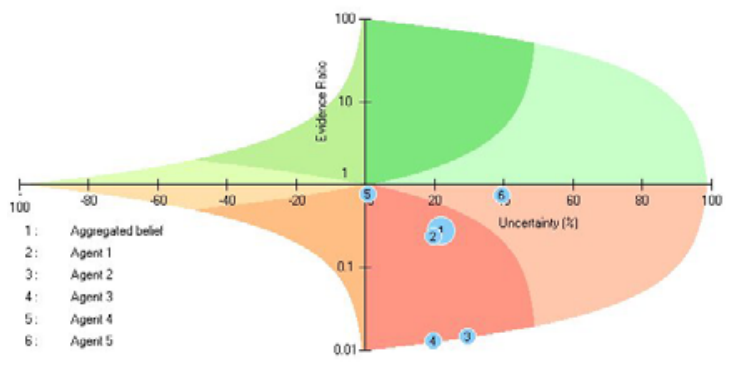

Figure II Ratio plot with respect to $H_{1}$

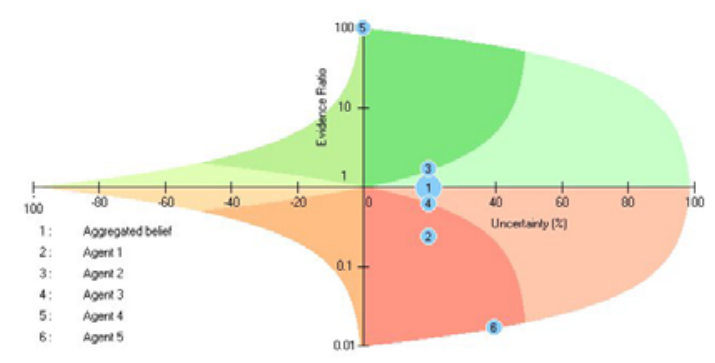

Figure III Ratio plot with respect to $\mathrm{H}_{2}$

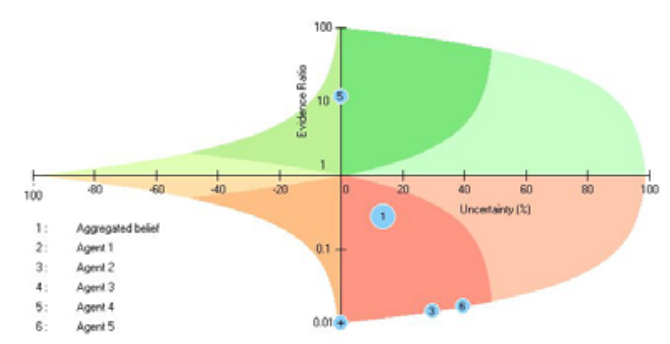

Figure IV Ratio plot with respect to $\mathrm{H}_{3}$

The weights assigned to the agents' beliefs have a significant influence on the aggregated results. This may reflect the power structure of the group. The agent with more power to make the final decision will be assigned a higher weight. Under a democratic mechanism, the weights can be evenly distributed among the agents. On the other hand, the weights are concentrated to just a few in an autocratic system. When the uncertainty value is low for each agent, this decision support approach acts like a voting mechanism.

Note that the scale of individual payoff or monetary values does not affect the group decision. Individual agents cannot manipulate the final decision by scaling up (or down) their payoffs. This ensures that each agent cannot influence the group decision more than their assigned weight.

\section{DISCUSSION AND FUTURE WORK}

In this paper, we have proposed a new decision support approach that can be used to make group decisions when risk, uncertainty, and conflicts of interest among stakeholders are involved. Based on evidence support logic and expected utility theory, this approach incorporates CBA within the framework of risk assessment. While this study makes a preliminary effort to link evidence support logic and economic analysis, it should be remembered that it has been conducted using some assumptions, for example, individual utilities on public welfare and independency of individual beliefs. So far we deal with group decision making as a static process. However, it is actually a dynamic process where individual beliefs may change along with interactions among agents and uncertainty may be reduced because of new information. Intelligent agents can learn in this process and be adaptive to the dynamics. Future research will focus on the dynamics in group decision making.

\section{REFERENCES}

[1] J. Adams, Risk, London: UCL Press, 1995.

[2] S. French, Decision theory: an introduction to the mathematics of rationality, Chichester: Ellis Horwood, 1986

[3] W. Feller, An introduction to probability theory and its applications, vol. 2, $2^{\text {nd }}$ ed. Wiley New York, 1971.

[4] W.Cui and D. I. Blockley, Interval probability theory for evidential support, International Journal of Intelligence Systems, Vol.5, No.2, pp.183-192. 1990.

[5] J. W. Hall, D. I. Blockley, and J. P. Davis, Uncertain inference using interval probability theory, International Journal of Approximate Reasoning, Vol.19, No.3-4, pp247-264, 1998.

[6] R. Pape, Developments in the tolerability of risk (TOR) and the application of ALARA, Nuclear Energy, Vol.36, No.6, pp.457-463, 1997.

[7] L. Foley, L. Ball, A. Hurst, J. Davis, and D. Blockley, Fuzziness, incompleteness and randomness classification of uncertainty in reservoir appraisal, Petroleum Geoscience, Vol.3, No.3, pp.203-209, 1997.

[8] J. W. Hall, D. I. Blockley, and J. P. Davis, A decision-support methodology for performance-based asset management, Civil Engineering and Environmental Systems, Vol.21, No.1, pp.51-75, 1998.

[9] J. Hey, and C. Orme, Investigating generalizations of expected utility theory using experimental data. Econometrica, Vol.62, No.6, pp. 1291-1326, 1994.

[10] C. Starmer, Developments in non-expected utility theory: the hunt for a descriptive theory of choice under risk. Journal ofEconomic Literature, Vol. 38, No.2, pp. 332-382, 2000.

[11] J. Li, S. Pollard, G. Kendall, E. Soane, G. Davies, Optimising risk reduction: an expected utility approach for marginal risk reduction during regulatory decision-making, Reliability Engineering \& System Safety, Vol.94, No.11, pp.1729-1734, 2009.

[12] K. Arrow and R. Lind, Uncertainty and the Evaluation of Public Investment decisions, American Economic Review, Vol. 60, No.3, pp.364-378, 1970.

[13] http://www.quintessa-online.com/TESLA/ESLGuide.pdf 